\title{
Enzyme kinetics at the molecular level
}

\author{
Arti Dua \\ Department of Chemistry, Indian Institute of Technology, Madras, Chennai-600036, India
}

\begin{abstract}
The celebrated Michaelis-Menten (MM) expression provides a fundamental relation between the rate of enzyme catalysis and substrate concentration. The validity of this classical expression is, however, restricted to macroscopic amounts of enzymes and substrates and, thus, to processes with negligible fluctuations. Recent experiments have measured fluctuations in the catalytic rate to reveal that the MM equation, though valid for bulk amounts, is not obeyed at the molecular level. In this mini-review, we show how new statistical measures of fluctuations in the catalytic rate identify a regime in which the MM equation is always violated. This regime, characterized by temporal correlations between enzymatic turnovers, is absent for a single enzyme and unobservably short in the classical limit.
\end{abstract}

\section{CLASSICAL ENZYME KINETICS}

Enzymes are biological catalysts that accelerate chemical reactions manyfold, without getting consumed in the catalytic process. Several biological processes involving the conversion of substrates to products, thus, rely crucially on the catalytic activity of enzymes. Specific enzymes control and regulate a wide-range of life-sustaining processes that vary from digestion, metabolism, absorption, blood clotting to reproduction. While specificity depends on detailed chemical structure of enzyme proteins, the rate at which enzymes carry out the catalytic conversion depends less on their chemical structure but more on physical parameters, including the amounts of enzymes, substrates, temperature, $\mathrm{pH}$, and so on [1]

In 1903, Victor Henri, in his doctoral thesis, studied the rate of hydrolysis of sucrose into glucose and fructose by the enzyme invertase, and laid the foundation for the understanding of enzymatic mechanisms from reaction rates [2]. In 1913, Leonor Michaelis and Maud Menten, building on the work of Victor Henri and many others, introduced the initial rate method for kinetic analysis. Using data for the initial rate of hydrolysis of sucrose, a simple reaction mechanism for enzyme catalyzed reactions, the Michaelis-Menten (MM) mechanism, was proposed [3] [5].

According to MM mechanism, enzyme E binds with substrate $\mathrm{S}$ to form an enzyme-substrate complex ES, which either dissociates irreversibly to form product $\mathrm{P}$, regenerating the free enzyme $\mathrm{E}$, or dissociates reversibly to release the substrate:

$$
E+S \stackrel{k_{1}}{\underset{k_{-1}}{\rightleftharpoons}} E S \stackrel{k_{2}}{\longrightarrow} P+E
$$

For thermodynamically large numbers of enzymes and substrates, deterministic mass action kinetics provides the rate equations for the MM mechanism in terms of the temporal variation of the concentrations of $E, E S$ and $P$. The mean rate of product formation $d[P] / d t$, then, quantifies the catalytic activity of enzymes through its dependence on the rate parameters for substrate binding $k_{1}[S]$, substrate release $k_{-1}$ and product formation $k_{2}$. Assuming a time scale separation in which the intermediate complex reaches a steady-state faster than the reactants and products, $d[E S] / d t \approx 0$, the mean rate of product formation yields

$$
V_{s s}=\frac{V_{\max }[S]}{[S]+K_{M}}
$$

the celebrated Michaelis-Menten (MM) equation, the steady-state "enzymatic velocity", which has widespread applicability in biochemical catalysis [6]. Here, $K_{M}=$ $\frac{k_{-1}+k_{2}}{k_{1}}$ is the Michaelis constant and $V_{\max }=k_{2}\left[E_{0}\right]$ is the maximum velocity at saturating substrate concentration, with $\left[E_{0}\right]$ being the initial enzyme concentration. The MM equation, in its double reciprocal form, $V_{s s}^{-1}=V_{\max }^{-1}+\frac{K_{M}}{V_{\max }}[S]^{-1}$, yields a linear variation of $V_{s s}^{-1}$ with $[S]^{-1}[7]$. Kinetic data for the variation of the initial rate of product formation with $[S]$, when fitted to this linear form, provides a simple way to estimate the rate parameters of several biochemical reactions. The hyperbolic dependence of the catalytic rate on substrate concentration, which the classical MM expression predicts, has had an enormous influence on the progress of biochemistry.

\section{ENZYME KINETICS AT THE MOLECULAR LEVEL}

Enzymatic reactions at the molecular level, however, do not proceed deterministically. Fluctuations of both quantum mechanical and thermal origin, termed as molecular noise, are inherent to reactions catalyzed by individual enzyme molecules. These impart stochasticity to each step in the MM mechanism, such that neither the lifetime of a given enzymatic state and nor the state to which it transits can be known with certainty. The effect of fluctuations, and thus the uncertainty, diminish progressively with increasing number of enzymes, and vanish for their macroscopic amounts. It is in the latter limit that the MM kinetics acquires its deterministic character and the classical description of enzymatic velocity as the "mean rate of product formation", is sufficient to characterize the catalytic activity of enzymes [8]. 
Biochemical catalysis, under physiological conditions, involves enzyme concentrations that are not thermodynamically large, but vary from nanomolar to micromolar. The substrates are typically between ten and ten thousand times more than the number of enzymes. At these low concentrations, termed as mesocopic, the presence of molecular noise is inherent to enzymatic reactions [9]. Once this fact is realized, a series of questions arise. Is classical enzymatic velocity, the MM equation, a valid description of the catalytic activity at physiologically relevant concentrations of enzymes? How do fluctuations in the rates of substrate binding, substrate release and product formation influence the rate of enzyme catalysis? How are uncertainties in the product formation times measured and characterized? What new information do these uncertainties carry about the catalytic mechanism that is lost in going over to the deterministic limit?

To address some of these questions, it is important to understand how single-enzyme kinetic data is collected and analyzed.

\section{A. Single-molecule kinetic data}

In the last two decades, advances in experimental techniques have finally made it possible to measure, with precision, the effect of molecular noise in enzyme catalyzed reactions, involving a single enzyme and numerous substrates [10, 11]. Such techniques have made it possible to monitor, in real time, the catalytic conversion of non-fluorescent substrates to fluorescent products, one substrate at a time, and yield a time series of the turnover times $T_{1}, T_{2}, \ldots$ for the first, second, ... product bursts. The turnover time series yields an equivalent series of the waiting times $\tau_{1}, \tau_{2}, \ldots$ between two consecutive product bursts $\tau_{p}=T_{p}-T_{p-1}$, with turnover number, $p=1,2, \ldots$.

A schematic of the MM mechanism for a single enzyme forming products in succession, one product per enzyme turnover, is shown in panel (a) of Fig. (1). The waiting and turnover times for $p=1,2,3, \ldots$ product formation are indicated as $\tau_{p}$ and $T_{p}$, respectively. Each waiting time is the sum of the lifetimes of $E$ and $E S$ states, $t_{E}$ and $t_{E S}, \tau_{p}=t_{E, j}+t_{E S, j}$, where the subscript index $j=1,2, \ldots$ denotes the number of times a given state $E$ or $E S$ is visited.

Due to the presence of molecular noise, the lifetimes of $E$ and $E S$ states, and hence $\tau_{p}$ and $T_{p}$, are random variables. Enzyme kinetics at the molecular level, thus, requires a stochastic description that includes intrinsic fluctuations in the lifetimes of $E$ and $E S$ states, the waiting times between two consecutive product turnovers, and the turnover times for $p$-th product formation. While the duration of time spent in $E$ and $E S$ states remains unobserved in single-molecule measurements, fluctuations in the lifetimes of these "hidden" states are "observed" as fluctuations in the product formation times $\tau_{p}$ and $T_{p}$.

To measure fluctuations in $\tau_{p}$, statistical measurements are carried out, in which waiting times for $p$-th product bursts are recorded, over and over again, for several stochastic realizations of the same reaction, under identical experimental conditions. A histogram of the recorded data is then used to extract probability distributions of waiting times, $w\left(\tau_{p}\right)$ and joint distributions of $p$-th and $q$-th waiting times, $w\left(\tau_{p}, \tau_{q}\right)$. Kinetic data at the molecular level is, thus, collected as waiting time distributions $w\left(\tau_{p}\right)$ and joint distributions $w\left(\tau_{p}, \tau_{q}\right)$, for given $p, q=1,2, \ldots, N$ and $[S]$. and analyzed in terms of their moments.

\section{B. Fluctuations in product formation times}

To understand how fluctuations in product formation times can arise, let us consider two stochastic realizations of the MM mechanism, termed as stochastic MM networks. Panels $(b)$ and $(c)$ of Fig. (1) illustrate two stochastic MM networks, each forming three products in succession, but following different pathways due to the presence of molecular noise. Stochasticity in these networks arises due to variation in the lifetimes of $E$ and $E S$ states, and the number of times a given state is visited. Both these aspects are included in the stochastic trajectories $(d)$ and (e), corresponding to (b) and (c) respectively, depicting the number of products $n_{p}$ formed in time $t$. Intrinsic fluctuations in $t_{E}$ and $t_{E S}$ are indicated as variation in the length of red and blue lines, and the number of times these colors switch between each other, before forming a product.

Panels (d) and (e) show that the first waiting time comprises of $\tau_{1}^{(b, c)}=t_{E, 1}+t_{E S, 1}$ for both (b) and (c). However, given that $t_{E}$ for $(b)$ is longer than $(c)$, with no variation in $t_{E S}$, implies $\tau_{1}^{(b)}>\tau_{1}^{(c)}$. This illustrates how stochasticity in the first waiting time can arise from variation in the lifetime of $E$ state. The waiting times for the second product burst are given by $\tau_{2}^{(b)}=t_{E, 1}+t_{E S, 1}+t_{E, 2}+t_{E S, 2}$ and $\tau_{2}^{(c)}=t_{E, 1}+t_{E S, 1}$. These captures stochasticity in $\tau_{2}$ due to fluctuations in the lifetime of $E$ and $E S$, and the number of times they switch between each other before forming a product. Together, they yield fluctuations in the number of products $n_{p}$ formed in a given time $t$, and the turnover time $T_{p}$ for the $p$-th product formation. These are indicated as dashed lines in panels (d) and (e).

\section{New statistical measures of fluctuations}

Single enzyme kinetic measurements yield waiting time distributions for product turnovers. A stochastic reformulation of the MM mechanism, presented in the next section, allows one to obtain these distributions theoretically. Enzyme kinetics at the molecular level can, then, be characterized in terms of the means and variances of these distribution for given $N$ and $[S]$. The first two mo- 

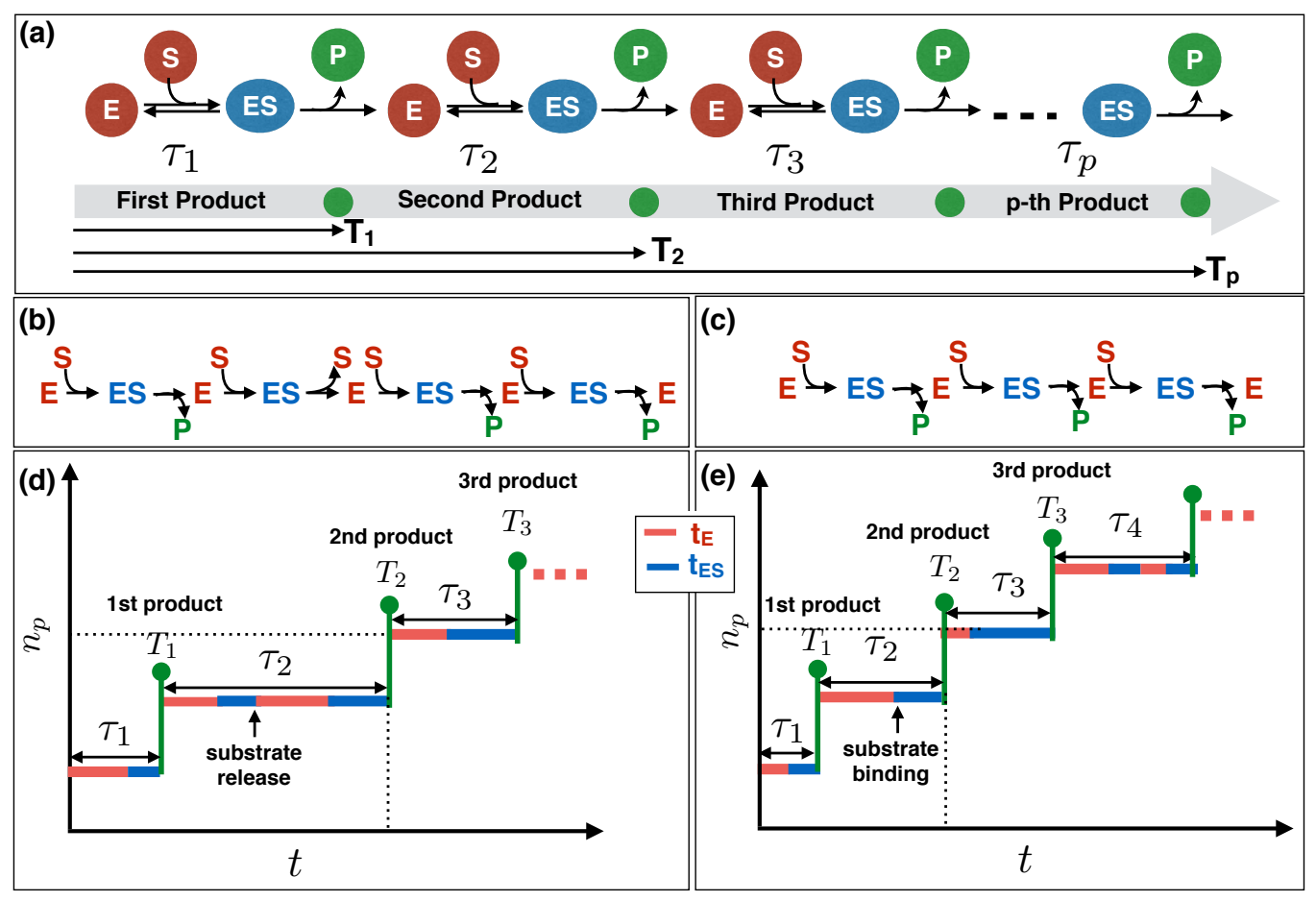

Figure 1. Panel (a) shows a schematic of the MM mechanism for a single enzyme forming products, one by one, in discrete turnover events. Here, $T_{p}$ with $p=1,2, \ldots$ is the $p$-th turnover time and $\tau_{p}=T_{p}-T_{p-1}$ is the waiting time between two consecutive product bursts. Panels (b) and (c) show two stochastic MM networks, each forming three products in succession, but following different pathways due to inherent stochasticity of the MM reaction at the molecular level. Panels (d) and (e) show two stochastic trajectories, corresponding to (b) and (c), for the number of products $n_{p}$ formed in time $t$. The lifetimes of $E$ and $E S, t_{E}$ and $t_{E S}$, are indicated by red and blue colors, respectively. Stochasticity in three consecutive waiting and turnover times is indicated by solid and dashed lines.

ments of $w(\tau)$, for instance, yields the expectation value $\langle\tau\rangle$, and variance $\sigma_{\tau}^{2}=\left\langle\tau^{2}\right\rangle-\langle\tau\rangle^{2}$ in the waiting time $\tau$. While the former is related to the mean catalytic rate of a single enzyme, the latter provides a new statistical measure of intrinsic temporal fluctuations in the catalytic rate [12, 13]. In particular, the dimensionless variance of the distribution, termed as the randomness parameter $r=\frac{\sigma_{\tau}^{2}}{\langle\tau\rangle^{2}}$, yields uncertainty in product formation times for a range of substrate concentrations [12 16.

The correlation, $C_{q}=\left\langle\delta \tau_{p} \delta \tau_{p+q}\right\rangle$, between a waiting time, $\tau_{p}$, and another, $\tau_{p+q}, q$ turnovers apart, where $\delta \tau_{p}=\tau_{p}-\left\langle\tau_{p}\right\rangle, p=1,2, \cdots$, provides another statistical measure of intrinsic temporal fluctuations that can be derived from the joint probability distribution of waiting times [9, 14]. A finite value of $C_{q}$ indicates that enzymatic turnovers are correlated in time, and the time duration of first turnover influences the time duration of subsequent turnovers. The correlations between enzymatic turnovers, thus, yield a "molecular memory" effect in which sequences of waiting times shorter or longer than the mean are more probable than sequences uniformly distributed about it [9, 14.

The landmark experiments on a single tetrameric enzyme, $\beta$-galactosidase, which is known to follow the MM equation in bulk amounts, reveal that the MM equation is violated at the molecular level 11. This effect due to molecular noise is linked to the simultaneous observation of $r>1$ and $C_{q}>0$. The magnitude of $r$ and the nature of decay of $C_{q}$ provide new fundamental constraints on potential enzymatic mechanisms that can be analyzed theoretically [9, 15, 16].

In the next section, we discuss the relevance of these statistical measures for the MM mechanism - a network with linear topology comprising of three reaction steps. We begin by presenting a stochastic reformulation of the MM kinetics for arbitrary number of enzymes [9, 14. An analysis of intrinsic temporal fluctuations, through the probability distributions of waiting times, is then presented to show how the statistical measures of intrinsic fluctuations - the randomness parameter and temporal correlations - carry chemically relevant information that is lost in passing to the deterministic limit. Specifically, the turnover kinetics of a single enzyme is compared with mesoscopic amounts of enzymes to show how deviations from the MM equation are inherently linked to fluctuations in product formation times. 



Figure 2. Single enzyme kinetics: Left panel shows waiting time distributions for $N=1$ enzyme for $p=1,10,100$ turnovers. The waiting times, irrespective of the turnover number, are identically distributed. Right panel shows that waiting time correlations between, first and $q=1,2, \ldots$ enzymatic turnovers are identically zero for all $[S]$. Single enzyme turnover kinetics follows a renewal stochastic process with independent and identically distributed waiting times.

\section{STOCHASTIC ENZYME KINETICS}

Stochastic enzyme kinetics includes inherent fluctuations at the molecule level by accounting for two important aspects of molecular noise. These include the discrete integer changes in the number of enzymes, complexes, products with time, and inherent stochastic character of each step of the MM mechanism. Both this aspects are included in the chemical master equation (CME) formalism of stochastic processes, which provides a general description for the turnover kinetics of $N$ discrete enzyme molecules [17, 18. So, while $N=1$ yields "rate equations" for a single enzyme [12, 13, the thermodynamic limit of $N \rightarrow \infty$ reduces the CME to a set of rate equations, governed by deterministic mass action kinetics [8]. In between the extremes of single enzyme $(N=1)$ and thermodynamic large $(N \rightarrow \infty)$ limits, the CME describes the turnover kinetics of mesoscopic enzyme concentrations.

The CME encodes mechanistic information from which waiting time distributions can be derived. The latter can be obtained from exact stochastic simulations of the CME [19. This involves generating a large number of stochastic trajectories of the MM mechanism, using data for the number of products versus time to obtain $\tau_{p}$ for $p=1,2, \ldots$, and histogramming this data to obtain $w\left(\tau_{p} ; N\right)$, for given $N$ and $[S]$. A similar procedure is followed to obtain the joint distribution of waiting times. The means and variances of these distributions provide kinetic measures that yield the enzymatic velocity and fluctuations in the catalytic rate for $N$ individual enzymes. These, for a single enzyme, are presented below.

\section{A. Single enzyme kinetics}

To describe the turnover kinetics of a single enzyme, we obtain waiting time distributions for first, second, third, ... product formation, corresponding to $p=1,2,3, \ldots$ turnovers, using exact stochastic simulations of the CME [9, 14. For this we generate typically $10^{6}$ stochastic trajectories of the MM mechanism for rate parameters $k_{a}=k_{2}=1$ and $k_{-1}=\frac{1}{2}$. The results are shown in Fig. (2).

The left panel of Fig. (2) shows the temporal variation of waiting time distributions for a single enzyme. The waiting times, irrespective of the turnover number $p$, are identically distributed. This implies that the distribution of waiting time between any two consecutive turnovers, for instance $w(\tau ; N=1)$, is sufficient to describe the turnover kinetics at the single enzyme level, and specification of the turnover index $p$ is not necessary. The right panel of Fig. (2) shows that waiting time correlations between enzymatic turnovers, $C_{q}$, are zero for all $q$ and $[S]$. Together, these results imply that waiting times are independently and identically distributed. The turnover kinetics of a single enzyme, thus, follows a renewal stochastic process with $\left\langle\tau_{p}\right\rangle=\langle\tau\rangle$ and $\left\langle T_{p}\right\rangle=p\langle\tau\rangle$ 9 .

Remarkably, the inverse of the mean waiting time of a single enzyme exactly recovers the MM equation, $\langle\tau\rangle^{-1}=\frac{k_{2}[S]}{[S]+K_{M}}$. Note, that the classical description of the steady-state enzymatic velocity is in terms of the rate of change of the mean number of products $V_{s s}=\lim _{t \rightarrow \infty} \frac{d\left\langle n_{p}(t)\right\rangle}{d t}=\frac{N k_{2}[S]}{[S]+K_{M}}$. From this, it follows that $V_{s s}=N\langle\tau\rangle^{-1}$. Using these identities, the classical steady-state enzymatic velocity can be reinterpreted as

$$
\frac{V_{s s}}{N}=\langle\tau\rangle^{-1}=\frac{p}{\left\langle T_{p}\right\rangle}=\frac{k_{2}[S]}{[S]+K_{M}}
$$

This is the single-enzyme analog of the classical MM equation, yielding a hyperbolic dependence of $\langle\tau\rangle^{-1}$ on substrate concentration [9, 14.

To quantify uncertainty in the waiting time $\tau$, the randomness parameter, $r=\frac{\left\langle\tau^{2}\right\rangle-\langle\tau\rangle^{2}}{\langle\tau\rangle^{2}}$, is evaluated from the mean and variance of $w(\tau ; N=1)$. This yields 

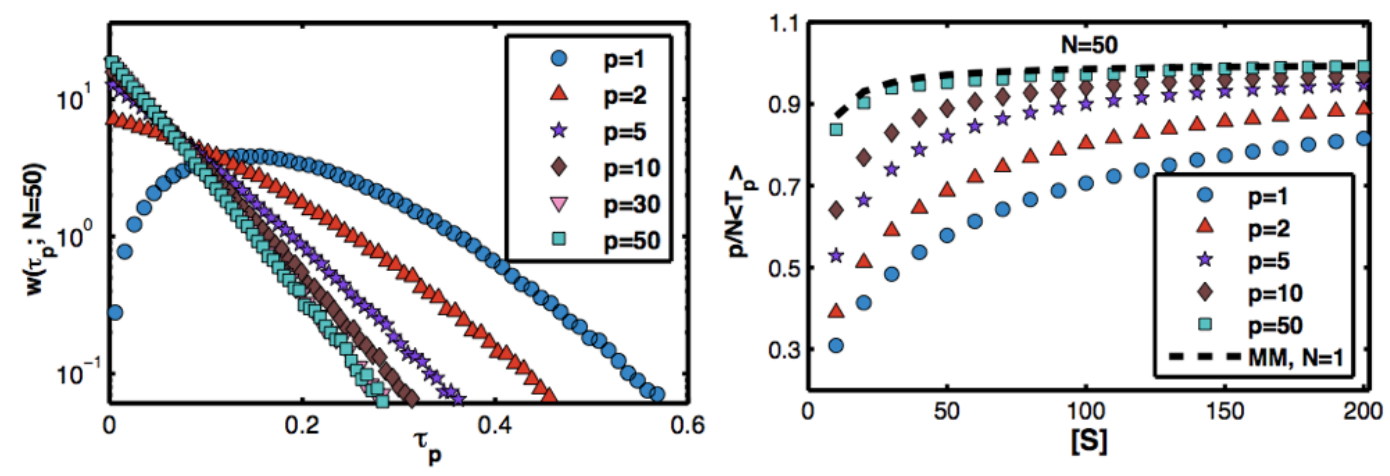

Figure 3. Enzyme kinetics at mesoscopic concentrations: Left panel shows waiting time distributions for $N=50$ enzymes for $p=1,2,5,10,30,50$ turnovers. The waiting times are non-identically distributed for $p \ll p^{*}$, the transient regime, but become identically distributed for $p \gg p^{*}$, the steady-state regime. Here, $p^{*}$ is the critical turnover number beyond which turnover time correlations decay. Right panel shows that the scaled enzymatic velocity $p / N\left\langle T_{p}\right\rangle$ for $N=50$ enzymes deviates from the MM equation in the transient regime, but converges to the MM equation in the steady-state regime.

$r<1$ for all $[S]$ [13]. This result is a specific case of a formal connection between the randomness parameter and network topology, which dictates that a reaction mechanism with $n$ sequentially connected kinetic states, $1 \stackrel{k}{\rightarrow} 2 \stackrel{k}{\rightarrow} 3 \stackrel{k}{\rightarrow} \cdots \stackrel{k}{\rightarrow} n \stackrel{k}{\rightarrow} n+1$, with exponentially distributed lifetime of each kinetic state, always yields $r=\frac{1}{n}$ [20]. Here, $n$ represents the number of rate determining steps in a linear reaction network. Thus, $r=\frac{1}{n}$ is the minimum amount of uncertainty that can be captured by the randomness parameter for networks with linear topologies. A generalization of this to linear networks, in which all (but the last) nearest neighbor transitions are reversible and occur with arbitrary rates, $1 \rightleftharpoons 2 \rightleftharpoons 3 \rightleftharpoons \cdots \rightleftharpoons n \rightarrow 1$, also yields $r<1$. Thus, irrespective of the number of kinetic intermediates, their connectivity with respect to each other, and rates of transition between neighboring states, $r \leq 1$ for all networks with linear topologies [15, [16.

The observation of $r>1$ in recent kinetic measurements on an MM enzyme, thus, rules out MM networks with linear topologies, and suggest that MM networks with branches need to be considered [13.

\section{B. Enzyme kinetics at mesoscopic concentrations}

In between the classical limit of thermodynamically large number of enzymes and a single enzyme, lies mesoscopic number of enzymes. To obtain waiting time distributions for mesoscopic concentrations, we carry out exact stochastic simulations of the CME [9, 14]. The results are presented in Figs. (3) and (4).

The left panel of Fig. (3) shows the temporal variation of waiting time distributions for increasing turnover numbers. The right panel shows, for the same turnover numbers, the variation of the enzymatic velocity of $N$ discrete enzymes, $\frac{p}{N\left\langle T_{p}\right\rangle}$, with substrate concentration. Interestingly, for turnover numbers much less than the critical turnover $p^{*}, p \ll p^{*}$, the waiting times are non-identically distributed and the MM equation is not obeyed. For $p \gg p^{*}$, the renewal turnover statistics and the MM equation are asymptotically recovered.

To understand this, the left panel of Fig. (4) shows the variation of waiting time correlations between enzymatic turnovers $C_{q}$ as a function of $q=1,2, \ldots$. For mesoscopic amounts of enzymes, temporal correlations between enzymatic turnovers, though appreciable for $p \ll p^{*}$, become negligible for $p \gg p^{*}$. Here, $p^{*}$ is the critical turnover number beyond with turnover time correlations decay.

Together, these results imply that the critical turnover number demarcates a transient regime $p \ll p^{*}$ from a steady state regime $p \gg p^{*}$ 14. In the transient regime, turnover kinetics is non-renewal, where waiting times are non-independent and non-identically distributed, and the MM equation is violated. In the steady-state regime, turnover kinetic acquire a renewal character and the MM equation is exactly recovered. The non-renewal nature of the turnover kinetics implies that $\left\langle T_{p}\right\rangle \neq p\left\langle\tau_{p}\right\rangle$. For mesoscopic amounts of enzymes, then, the steady-state enzymatic velocity can be reinterpreted as

$$
V_{s s}=\lim _{p \rightarrow \infty} \frac{p}{\left\langle T_{p}\right\rangle}=\frac{N k_{2}[S]}{[S]+K_{M}} .
$$

Note that the above expression is only valid for $p \gg p^{*}$, where $C_{q}=0$.

For the MM mechanism, the waiting times between enzymatic turnovers are anti-correlated, $C_{q}<0$. This implies that a long (or short) first waiting time (compared to its mean value) is more likely to be followed by a short (or long) second waiting time. This memory effect, is shown as the heat map of joint probability distribution $w\left(\tau_{1}, \tau_{2}\right)$ in Fig. (4) inset. 

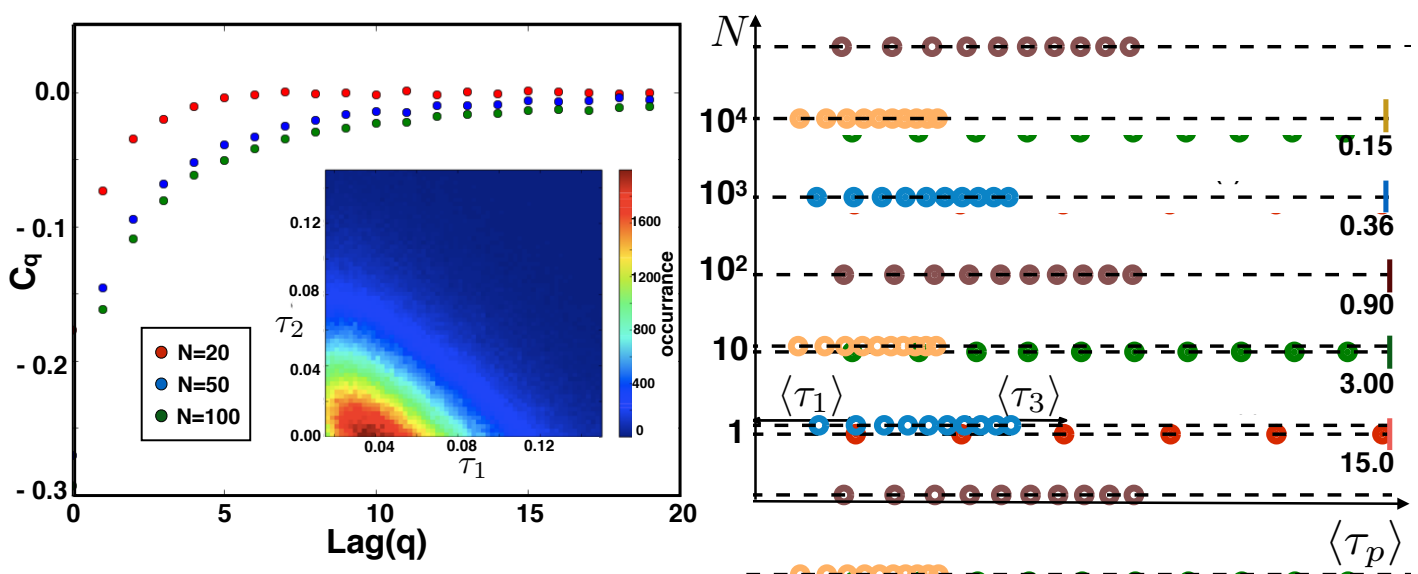

Figure 4. Left panel shows temporal correlations between $\tau_{1}$ and $\tau_{q}$ with $q=1,2, \ldots$ waiting times. The waiting times are anticorrelated, where a short (or long) first waiting time is more likely to be followed by a long (or short) second waiting time (inset). This memory effect, though stronger and short lived for smaller $N$, becomes weaker and long lived for larger $N$. Right panel shows the variation of $\left\langle\tau_{p}\right\rangle$, for first ten turnovers $p=1,2, . .10$ with increasing $\mathrm{N}$. The discrete turnover events acquire a deterministic character, with the increase in $N$, as waiting times become infinitesimally short and products appear to form continuously in time.

\section{The classical limit from molecular perspective}

To understand how the classical limit of enzyme kinetics can emerge from molecular kinetics, we show in the right panel of Fig. (4), the variation of $\left\langle\tau_{p}\right\rangle$ for first ten product turnovers, as a function of increasing $N$. For $N=1$, since $C_{q}=0$, the mean waiting times are identical $\left\langle\tau_{p}\right\rangle=\langle\tau\rangle$, and the MM equation, $\langle\tau\rangle^{-1}=\frac{k_{2}[S]}{[S]+K_{M}}$, is always obeyed.

For $N>1$, there emerge two kinetic regimes - a transient regime for $p \ll p^{*}$, and a steady-state regime for $p \gg p^{*}$. In the transient regime, non-identical mean waiting times $\left\langle\tau_{p}\right\rangle$ become progressively shorter with increasing $N$ and $p$. In the steady-state regime, similarly, there is an $N$-fold decrease in the mean waiting time $\langle\tau\rangle$. The discrete turnover events acquire a deterministic character, with the increase in $N$, as waiting times between consecutive products become shorter, and the number of products in a small time interval become progressively larger.

In the limit of macroscopic amounts of enzymes $N \gg$ 1 , thus, waiting times become infinitesimally small, products appear to form continuously in time, and the transient regime becomes too short to be experimentally accessible. In this classical limit, kinetic data for the "initial" mean rate of product formation yields the steadystate enzymatic velocity. From the molecular perspective, the absence of temporal correlations between enzymatic turnovers in the steady-state regime, implies that the MM equation is obeyed.

\section{CONCLUSIONS}

In this mini-review, a stochastic reformulation of the MM kinetics for a single and mesoscopic amounts of enzyme(s) has been presented. An analysis of intrinsic temporal fluctuations, through the probability distributions of waiting times, shows that the MM equation is always violated in the transient regime. This regime, characterized by temporal correlations between enzymatic turnovers, while absent for a single enzyme, is unattainable in the classical limit. In both these limits, thus, the MM equation, is exactly obeyed.

At mesoscopic concentrations, the transient regime is observably large, and temporal correlations between enzymatic turnovers yields a molecular memory effect in which the time duration of the first turnover influences the time duration of subsequent turnovers. In the presence of molecular memory, the MM equation is not obeyed at the molecular level. This effect, due to molecular cooperativity, leads to a slowing down of the MM kinetics in the transient regime. The critical turnover time beyond which the correlation decays and the molecular memory fades marks the time scale for the crossover from a transient regime where the MM equation is violated, to a a steady-state regime where the MM equation is recovered exactly at the molecular level.

From these general results, we conclude that for mesoscopic amounts of enzymes, there emerges a transient regime in which enzymatic turnovers are correlated in time, and the the MM equation, derived using time scale separation, is always violated.
[1] Athel Cornish-Bowden, Fundamentals of Enzyme Kinetics, Vol.510, Wiley-Blackwell Weinheim, Germany, 2012.
[2] V Henri, Theorie Generale delaction de Quelques Di- 
astases, C.R.Hebd. Seances Acad. Sci., 1902, Vol.135, pp.916-919, 1902.

[3] Athel Cornish-Bowden, One Hundred Years of Michaelis-Menten Kinetics, Perspectives in Science, Vol.4, pp.3-9, 2015.

[4] Ute Deichmann, Stefan Schuster and Jean-Pierre Mazat, Commemorating the 1913 Michaelis-Menten paper die kinetik der invertinwirkung: three perspectives, The FEBS Journal, Vol.281, No.2, pp.435-463, 2014.

[5] L Michaelis and M L Menten, Die Kinetik der Invertinwirkung, Biochem.Z., Vol.49, pp.333-369, 1913.

[6] Felix Wong, Annwesha Dutta, Debashish Chowdhury and Jeremy Gunawardena, Structural Conditions on Complex Networks for the Michaelis-Menten input-output response, Proceedings of the National Academy of Sciences, Vol.115, No.39, pp.9738-9743, 2018.

[7] Hans Lineweaver and Dean Burk, The Determination of Enzyme Dissociation Constants, Journal of the American chemical society, Vol.56, No.3, pp.658-666, 1934.

[8] Anthony F Bartholomay, A Stochastic Approach to Statistical Kinetics with Application to Enzyme Kinetics, Biochemistry, Vol.1, No.2, pp.223-230, 1962.

[9] Soma Saha, Somdeb Ghose, R Adhikari Arti Dua, Nonrenewal Statistics in the Catalytic Activity of Enzyme Molecules at Mesoscopic Concentrations, Physical review letters, Vol.107, No.21, p.218301, 2011.

[10] Brian P English, Wei Min, Antoine M Van Oijen, Kang Tack Lee, Guobin Luo, Hongye Sun, Binny J Cherayil, SC Kou, and X Sunney Xie, Ever-fluctuating single enzyme molecules: Michaelis-menten equation revisited, Nature chemical biology, Vol.2, No.2, pp.87-94, 2006.

[11] W Min and B P English, G Luo, B J Cherayil, S C Kou and X S Xie, Fluctuating Enzymes: Lessons from Single-
Molecule Studies, Acc. Chem. Res., Vol.38, pp.923-931, 2005.

[12] Soma Saha, Antara Sinha and Arti Dua, Single-molecule Enzyme Kinetics in the Presence of Inhibitors, The Journal of chemical physics, Vol.137, No.4, p.045102, 2012.

[13] Ashutosh Kumar and Hiranmay Maity and Arti Dua, Parallel versus Off- Pathway Michaelis-Menten Mechanism for Single-Enzyme Kinetics of a Fluctuating Enzyme, The Journal of Physical Chemistry B, Vol.119, No.27, pp.8490- 8500, 2015.

[14] Ashutosh Kumar, R Adhikari and Arti Dua, Kumar, Adhikari, and Dua Reply, Phys. Rev. Lett., Vol.119, No.9, p.099802, August 2017.

[15] Jeffrey R Moffitt and Carlos Bustamante Extracting Signal from Noise: Kinetic Mechanisms from a Michaelis-Menten-like expression for enzymatic fluctuations, The FEBS journal, Vol.281, No.2, pp.498-517, 2014.

[16] Jeffrey R Moffitt, Yann R Chemla and Carlos Bustamante, Mechanistic Constraints from the Substrate Concentration Dependence of Enzymatic Fluctuations, Proceedings of the National Academy of Sciences, Vol.107, No.36, pp.15739-15744, 2010.

[17] Crispin W Gardiner, Handbook of Stochastic Methods for Physics, Chemistry and the Natural Sciences, Vol.13 of Springer Series in Synergetics, 2004.

[18] Nicolaas Godfried Van Kampen, Stochastic Processes in Physics and Chemistry, Vol.1, 1992.

[19] Daniel T Gillespie, Stochastic Simulation of Chemical Kinetics, Annu. Rev. Phys. Chem., Vol.58, pp.35-55, 2007.

[20] Aldous David and Shepp Larry, The Least Variable Phase Type Distribution is Erlang, Communications in Statistics. Stochastic Models, Vol.3, No.3, pp.467-473, Taylor \& Francis, 1987. 